\title{
Visuelle Politik: Perspektiven eines politikwissenschaftlichen Forschungsbereichs
}

\author{
Petra Bernhardt ${ }^{1,}$, , Karin Liebhartt, ${ }^{1, * *} \&$ Andreas Pribersky ${ }^{1, * * *}$ \\ 1 Institut für Politikwissenschaft, Universität Wien \\ * petra.bernhardt@univie.ac.at \\ **karin.liebhart@univie.ac.at \\ **andreas.pribersky@univie.ac.at
}

\section{Zusammenfassung}

Der Beitrag beschäftigt sich mit Visueller Politik, einem interdisziplinär ausgerichteten Forschungsbereich der Politikwissenschaft. Anhand eines Fotos von US-Präsident Donald J. Trump, das am 15. Jänner 2019 während eines Empfangs im Weißen Haus aufgenommen wurde und das sowohl in den USA als auch international für mediale Aufmerksamkeit und Anschlusskommunikation in Sozialen Netzwerken gesorgt hat, zeigt der Beitrag, durch welche Prämissen und Analyseperspektiven der Forschungsbereich Visuelle Politikgekennzeichnet istundwieeinebildkompetente Politikwissenschaft auf die Herausforderungen einer professionalisierten politischen Kommunikation reagieren kann.

\section{Schlüsselwörter}

Visuelle Politik, Visuelle Politische Kommunikation, Visuelle Analysen, Visuelle Kompetenz / Visuelle Literalität

\section{Visual Politics: Perspectives of a Research Field in Political Science}

\begin{abstract}
The article addresses visual politics as an interdisciplinary research area in political science. Using a photo of US president Donald J. Trump, which was taken during a reception at the White House on 15 January 2019 and which attracted media attention and follow-up communication in social networks both in the USA and internationally, the article shows the premises and analytical perspectives that characterise the research field of visual politics. Hence, it demonstrates how a visually competent approach in political science can respond to the challenges posed by professionalised political communication.
\end{abstract}

\section{Keywords}

Visual Politics, Visual Political Communication, Visual Analysis, Visual Competence / Visual Literacy

The authors have declared that no competing interests exist. 


\section{Einleitung}

Am I5. Jänner 2019 gingen Fotos und Videoaufnahmen aus dem Weißen Haus um die Welt. Das Bildmaterial zeigt den amtierenden US-Präsidenten Donald J. Trump während eines Empfangs der Footballmannschaft Clemson Tigers, dem Sieger der College Football Playoffs. Trump präsentiert sich dabei unter anderem im State Dining Room des Weißen Hauses hinter einer mit Kandelabern und Silberbesteck gedeckten Tafel, auf der sich verpacktes Fast Food stapelt (siehe Abb. I). Die ungewöhnliche Inszenierung wurde von einer Pressekonferenz mit Photo Opportunity begleitet. Trump nutzte die Gelegenheit nicht nur für einen Fototermin, sondern auch für einen Seitenhieb auf den politischen Mitbewerber: Der Government Shutdown und die damit verbundene Schließung der Küche des Weißen Hauses habe ihn gezwungen, seine Gäste mit Fast Food zu bewirten, das er aus der eigenen Tasche bezahlt habe. Was folgte war eine enorme nationale und internationale Medienresonanz auf ein Ereignis, dessen Bedeutung die Aufmerksamkeit zunächst keineswegs nahe legt.

Abbildung 1: Donald J. Trump im State Dining Room des Weißen Hauses während eines Empfangs der Clemson Tigers am 15.01.2019.

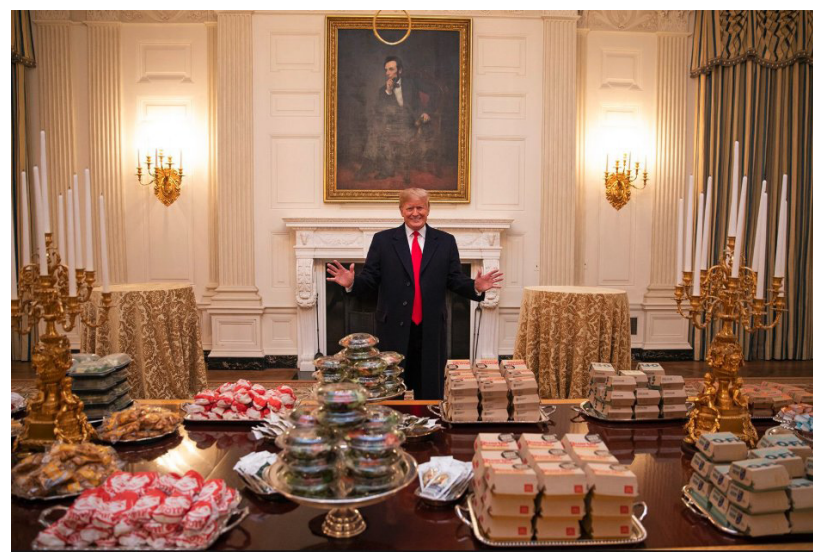

Official White House Photo von Shealah Craighead, veröffentlicht auf dem offiziellen Twitter-Account des Weißen Hauses (https://twitter.com/WhiteHouse/status/1084988299041738752).

Bildmaterial wie jenes aus dem State Dining Room stellt heute einen wichtigen Bestandteil der strategischen Kommunikation der Spitzenpolitik dar. Fotos der Veranstaltung wurden über den offiziellen Twitter-Account des Weißen Hauses verbreitet (The White House 20I9a). Videos wurden unter anderem auf der offiziellen Facebook-Seite des Weißen Hauses (The White House 20I9b) verfügbar gemacht und auf dem Twitter-Account von Pressesprecherin Sarah Sanders (Sanders 2019) gepostet. Darüber hinaus wurde das Bildmaterial zum Gegen- stand breiter medialer Anschluss- und Meta-Kommunikation. Am Tag nach der Veranstaltung berichteten zahlreiche nationale und internationale Medien (z. B. The New York Times, The Guardian, BBC, Süddeutsche Zeitung, u. a.) über den Fast Food Empfang im Weißen Haus. Auch in Sozialen Netzwerken wurde das Bildmaterial zum Gegenstand unterschiedlicher Aneignungsformen, die von satirischen Bildbearbeitungen bis zu kritischen Kommentaren reichten. Der Erfolg des Fotos in klassischen Medien und Sozialen Netzwerken legt eine Reihe von Fragen nahe: Wie gelingt es politischen AkteurInnen, mit Bildmaterial öffentliche Aufmerksamkeit zu erregen? Was wird gezeigt, wie wird es gezeigt und welche strategischen Entscheidungen werden dabei sichtbar? Welche Spuren legt das Bild, auf welche anderen Bilder verweist es? Welche Deutungsangebote legt das Foto nahe und welche bildimmanenten Faktoren könnten für die Verbreitung des Fotos verantwortlich sein? Welche Interpretationen erfährt das Bildmaterial im Rahmen medialer Anschlusskommunikation und einer Verbreitung in Sozialen Netzwerken? Welche Schlüsse lassen sich aus der Beantwortung dieser Fragen für die Analyse zeitgenössischer politischer Kommunikation ziehen?

Dieser Beitrag nimmt das Foto von Donald J. Trump deshalb zum Ausgangspunkt einer Diskussion über Visuelle Politik als Forschungsbereich der Politikwissenschaft. Er beschäftigt sich mit der Frage, welche unterschiedlichen Funktionen Bildmaterial im Rahmen strategischer politischer Kommunikation, politischer Auseinandersetzungen sowie im Rahmen öffentlicher und medialer Debatten haben und wie die Politikwissenschaft darauf reagieren kann. Wenngleich der Beitrag mit Bezugnahmen auf rezente Forschungsarbeiten im Bereich der Politik- und Kommunikationswissenschaft argumentiert, stellt er keinen Anspruch auf einen vollständigen Forschungsüberblick im Sinne einer MetaAnalyse, sondern versteht sich dezidiert als Diskussionsbeitrag. Der Beitrag beginnt mit einer Darstellung zentraler Prämissen des Forschungsbereichs, setzt fort mit der Diskussion verschiedener Analyseperspektiven entlang einer Einordnung des Trump-Fotos und schließt mit einer Diskussion der Bedeutung Visueller Kompetenz für die Politikwissenschaft.

\section{Prämissen Visueller Politik}

Bilder spielen im sogenannten „visual age” eine essentielle Rolle bei der kommunikativen Konstruktion sozialer Wirklichkeit (Lobinger 2015, 9I). Die sozialwissenschaftliche Beschäftigung mit visuellen Phänomenen hat seit den I990er-Jahren dementsprechend stetig zugenommen. Die Frage, wie soziale AkteurInnen mit Bildern handeln, Sinn erzeugen und Fakten schaffen, ist zu einem wichtigen Thema insbesondere soziologischer, 
anthropologischer und kommunikationswissenschaftlicher Forschung geworden. Mit dieser wissenschaftlichen Orientierung, die in der einschlägigen Literatur unter Verwendung der Begriffe "pictorial turn“ (Mitchell 1992) und „iconic turn“ (Boehm I994) als eine Wende zum Bild beschrieben wurde, ist die Annahme verbunden, dass Visualität eine zunehmende Bedeutung in der gegenwärtigen Produktion und Verbreitung von Wissen zukommt.

In der Politikwissenschaft findet diese Entwicklung einer stärkeren Orientierung an visuellen Phänomenen mit Verzögerung statt. Das liegt vor allem an dem Umstand, dass in der Disziplin tendenziell eine kritische Perspektive auf medienzentrierte Politikherstellung und -darstellung vorherrscht, die unter Begriffen wie „Mediokratie“ (vgl. Meyer 200I und 2010), „Politainment” (vgl. Dörner 200I) und Konzepten wie "Mediatisierung" (vgl. Riedl/Mauer/Mühl 20I8) diskutiert wird. Strömbäck (2008) stellt fest, dass im Zuge des Prozesses der zunehmenden Mediatisierung von Politik politische AkteurInnen die „Medienlogik” in einem Ausmaß adoptieren, dass diese die politische Handlungslogik schlussendlich substituiert. Diese kritische Auslegung eines Wechsels zu Mediendemokratien (vgl. Jarren/Donges 20II) bezeichnet spätestens seit dem Beginn des letzten Jahrzehnts eine weitgehende Ausrichtung der Politik an medialen Logiken, Themenstrukturen, Präsentationsformen und Aufmerksamkeitsmechanismen, wodurch die Politik Gefahr laufe, zu einer Scheinpolitik zu wer-

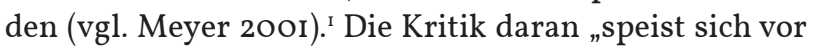
allem aus der Visualität diagnostizierter Symptome: symbolische Schein-, Image- und Eventpolitik“ (Grittmann 2009, 34).

Die Kommunikationswissenschaftlerin Elke Grittmann weist darauf hin, dass dadurch in der Debatte die grundlegende Frage aus dem Auge verloren wird, „warum welches politische Angebot wie überhaupt öffentlich sichtbar wird" (ebd.). Diese Perspektive ist inhärent machtpolitisch und macht Visualität als eine zentrale Ressource politischen Handelns erkennbar: „Politische Prozesse haben eine visuelle Dimension - und zwar nicht nur ausnahmsweise, sondern regelmäßig" (Drechsel 2009, 159). Der Verweis auf die „Regelmäßigkeit” umfasst auch die historische Bedeutung visueller Repräsentation: Der Historiker und Kulturwissenschaftler Peter Burke hat diese etwa an der Wirkung der vielfältigen zeitgenössischen Darstellungen des französischen Sonnenkönigs Ludwig XIV. im I7. und beginnenden I8. Jahrhundert für dessen politische Bedeutung und seinen bis heute bestehenden Nachruhm rekonstruiert (Burke 1992).

I Riedl/Maurer/Mühl (2018, I4) ziehen aus der Analyse empirisch erhobener Daten allerdings auch den Schluss, dass für politische AkteurInnen „die in der politischen Kommunikationsforschung als allgegenwärtig angesehene Medienlogik" keine Konstante bildet, sondern auf der individuellen Ebene in unterschiedlich ausgeprägtem $\mathrm{Ma}$ adaptiert und adoptiert wird - am intensivsten in den Bereichen Dramatisierung und Konflikthaftigkeit (ebd., I2).
Diese visuelle Dimension des Politischen wird zwar immer wieder thematisiert (zuletzt etwa ausführlich in Bleiker 20I8). Ihr Stellenwert und ihre Bedeutung für politikwissenschaftliche Fragestellungen und Analysen konnten damit bisher aber nur ungenügend vermittelt werden:

„Images surround everything we do. This omnipresence of images is political and has changed fundamentally how we live and interact in today's world. Scholarly fields such as art history and media, cultural and communication studies have for long examined visual representations. But we still know far too little about the precise role visuality plays in the realm of politics and international relations. And we know even less about the concrete practical implications" (Bleiker 20I8, I).

Diese selektive Rezeption in der aktuellen politikwissenschaftlichen Forschung steht im Gegensatz zu wesentlichen Aspekten ihrer traditionellen Fragestellungen und Argumentationslinien: als Beispiel dafür soll hier nur auf die Arbeiten zweier Vertreter der Gründergeneration der Disziplin im deutsch(sprachig)en Raum nach 1945 verwiesen werden: Zum einen auf Arbeiten von Dolf Sternberger, der u. a. die Politische Vierteljahrsschrift, die offizielle Zeitschrift der Deutschen Vereinigung für Politikwissenschaft, begründet hat: Sternberger hat, über seine politikwissenschaftlichen Arbeiten hinaus, bereits 1957 - lange vor einer breiten öffentlichen Wiederentdeckung dieser Stilrichtung - einen Essay Über den Jugendstil als ästhetische und soziale Bewegung veröffentlicht. Und er hat sein Bildwissen $\mathrm{zu}$ politikwissenschaftlichen Analysen genutzt, etwa in seinem Essay über Herrschaft und Vereinbarung (1980). ${ }^{2}$ Klaus von Beyme - der u. a. bei Sternberger in Heidelberg Politikwissenschaft studierte - hat ebenfalls eine Reihe kunstgeschichtlicher Arbeiten, vor allem zur Architekturgeschichte, publiziert. ${ }^{3}$ Beide Autoren haben mit ihren Arbeiten im Bereich der Visuellen Politik den Anspruch verbunden, Beiträge zu grundlegenden Fragen der Politischen Theorie bzw. der historischen ebenso wie der zeitgenössischen Repräsentation von Politik zu leis-

2 In dieser Analyse der historischen Ursprünge der Ausdifferenzierung zwischen Formen autoritären und demokratischen Regierens nimmt Sternberger einen Vergleich des Frontispiz von Hobbes' Leviathan mit den Schwurbrüdern des Schweizer Bundes zum Ausgangspunkt.

3 Dazu zählen Werkportraits von Architekten wie Roland Rainer oder eine vergleichende Studie des Wiederaufbaus in den beiden Deutschen Staaten nach 1945 (von Beyme 1987), in denen von Beyme sich auch mit der Problematik des Verhältnisses von totalitären und demokratischen Bauformen auseinandersetzt. Beyme hat darüber hinaus Arbeiten zur politischen Programmatik von Architektur und Kunst (z.B. von Beyme 2005) verfasst bzw. herausgegeben, deren Schwerpunkt die Fragen der Repräsentation des Politischen in der bildenden Kunst und deren Einfluss auf die Politisierung von Gesellschaften und den öffentlichen Raum bilden. 
ten: dieser Ansatz ist in ihrem Verständnis der zentralen Rolle visueller Phänomene für Wahrnehmung und Sichtbarkeit als Voraussetzungen politischer Bedeutung begründet. Eine Weiterführung dieser Ansätze für das Verhältnis von Urbanismus und Politik findet sich etwa bei Wilhelm Hofmann (2OII), für die Analyse der Wirksamkeit politischer Ideen und Persönlichkeiten ist dieser Ansatz u.a. von Herfried Münkler wieder aufgegriffen worden (Münkler/Hacke 2009). Methodologisch orientieren sich die hier erwähnten Untersuchungen ebenso wie die übrigen Werke Sternbergers oder von Beymes zur Visuellen Politik an einem interdisziplinären, mit kulturwissenschaftlichen Analysemethoden und Fragestellungen arbeitenden Forschungskonzept. Bei den beiden Autoren hatte diese Interdisziplinarität in deren Bildungswegen eine persönliche Grundlage für die Verbindung unterschiedlicher Disziplinen, die sie zur Formulierung von Fragestellungen im Feld der Visuellen Politik bzw. zu dieser als Referenzrahmen des Politischen geführt hat. Forschungen im Bereich der Visuellen Politik zeichnen sich im Allgemeinen durch eine interdisziplinäre Orientierung aus und knüpfen an unterschiedliche Traditionslinien in den Geistes-, Kultur- und Sozialwissenschaften an. Disziplinäre Bezugspunkte finden sich unter anderem in den Medien- und Kommunikationswissenschaften, in der Visual History, in der Kunstgeschichte, in den Bildwissenschaften und in den Visual Culture Studies.

Wenn der vorliegende Beitrag die Frage beantworten möchte, wie eine spezifisch politikwissenschaftliche Form Visueller Kompetenz aussehen könnte, ist zunächst die Frage nach dem Gegenstandsbereich Visueller Politik zu klären. Für die Formulierung eines Bildbegriffs Visueller Politik sind drei Überlegungen zentral: Erstens geht es um die Frage, um welche Bilder sich Visuelle Politik kümmern sollte. Der Politikwissenschaftler Benjamin Drechsel hat vorgeschlagen, politische Bilder nicht über ihre Gattung (z.B. politische Plakate, politische Karikaturen, etc.) zu definieren, sondern über ihre Funktion. Für Drechsel kann jedes Bild zum politischen Bild werden, „wenn es durch die entsprechende Verwendung, Fragestellung oder Interpretation politisch kontextualisiert wird“ (Drechsel 2007, III). Das bedeutet, dass Bilder nicht „an sich politisch [sind], sondern stets im Rahmen dynamischer Vorgänge (ent-)politisiert werden“ (ebd.). Diese Perspektive rückt Fragen nach öffentlichen Sichtbarkeiten und Unsichtbarkeiten und den damit verbundenen Machtfragen sowie den Eingriff von Bildmedien in politische Prozesse ins Zentrum der Aufmerksamkeit (vgl. ebd., II6).

Zweitens ist zu klären, was das Objekt „Bild“ im Bereich Visueller Politik ausmacht. Die nur scheinbar banale Frage "Was ist ein Bild?" wird in unterschiedlichen wissenschaftlichen Traditionen unterschiedlich beantwortet. Die im Englischen gängige, wenngleich nicht im- mer konsequent eingehaltene Unterscheidung zwischen "pictures" als materielle und "images" als immaterielle Bilder lässt sich im Deutschen aufgrund fehlender Begrifflichkeiten nicht anwenden (vgl. Müller 2009, 9). Im Deutschen schließt der Begriff "Bild“ sowohl innere als auch äußere Bilder mit ein (vgl. Boehm 2007, II). Materielle Abbilder können als Quellen für die Rekonstruktion von Denkbildern bzw. mentalen Bildern dienen (vgl. Müller 2003, 2off). Abbilder bringen Denkbilder hervor, umgekehrt bedeutet das aber nicht, dass sich jedes Denkbild in Form eines Abbilds manifestiert (Müller/ Geise 2015, 2I). Für die Untersuchung des Zusammenhanges zwischen politischen Bildern und den dadurch beförderten "Images“" politischer AkteurInnen ist diese Unterscheidung zentral: „Als mentales Bild ist ein Image vor allem eine visuelle Vorstellung" (ebd., 35, Herv. i.O.).

Drittens ist festzuhalten, dass Visuelle Politik ihr Erkenntnisinteresse auf visuelle Phänomene als Quellenmaterial richtet. Bilder dienen der Visuellen Politik nicht zur Veranschaulichung oder Illustration, um Argumente zu untermauern, die sich auch sprachlich zum Ausdruck bringen lassen, sondern vielmehr als Analysegegenstand, der Zugang zu sozialwissenschaftlich relevanten Informationen ermöglicht. Visualisierungen stehen nicht in einem schlichten Abbildungsverhältnis zur Wirklichkeit, sondern sind das Ergebnis kulturell geprägter Darstellungs- und Rezeptionsweisen, die Veränderungen unterliegen. In seiner Verbindung ikonologischer und ideologiekritischer Analysen plädiert der Bildtheoretiker W.J.T. Mitchell für eine Untersuchung von Bildern in ihren soziokulturellen Kontexten (Mitchell 1992) und für ein Hinterfragen des spezifischen Verhältnisses zwischen "pictures" und "images" (Mitchell 1990).

Visuelle Politik beschäftigt sich also mit materiellen Bildern und Images, die eine politische Kontextualisierung erfahren. Politische Bildlichkeit lässt sich daher nicht ontisch vorweg definieren, sondern wird durch den Verwendungszusammenhang bestimmt. Politische Bilder werden deshalb im Kontext ihrer Verwendung als Quellenmaterial der Visuellen Politik erhoben: relevant sind dabei sowohl das Bildhandeln, als auch das Herstellen von Bildern zu politischen Zwecken, nicht zu Zwecken der Illustration oder Veranschaulichung. In den folgenden Abschnitten werden verschiedene Analyseperspektiven Visueller Politik mit Bezug auf das Foto aus dem State Dining Room skizziert, die eine Rekonstruktion des Bildhandelns und des Herstellens von Bildern ermöglichen.

\section{Ein Trumpsches Spektakel: Visuelle Politische Kommunikation und Politische Inszenierung}

Das Foto aus dem State Dining Room des Weißen Hauses ist zunächst ein Bestandteil strategischer politischer 
Kommunikationsaktivitäten Donald J. Trumps. Es wurde im Rahmen einer vorab definierten Photo Opportunity im Weißen Haus aufgenommen, bei der nicht nur JournalistInnen, sondern auch die persönliche Fotografin des Präsidenten, Shealah Craighead, anwesend waren. Das dabei entstandene Bildmaterial wurde für die weitere Nutzung auf offiziellen Social Media Accounts ausgewählt (z.B. dem Twitter-Account des Weißen Hauses) und damit als für das Ereignis repräsentatives Bildmaterial autorisiert. Es entspricht damit der in der Visuellen Kommunikationsforschung gängigen wertneutralen Definition von Inszenierung als „öffentliche Zurschaustellung...einer Person" (Müller/Geise 2015, 32) im Sinne „bewusst gestaltete(r) Realität” (ebd., 34). Dieser Entstehungskontext des Bildmaterials ist für seine weitere Einordnung relevant: als persönliche Fotografin hat Shealah Craighead die Aufgabe, Donald Trump in einer für ihn möglichst vorteilhaften Situation im Sinne der $\mathrm{zu}$ vermittelnden Botschaft zu fotografieren. Ihre PRorientierte Arbeit unterscheidet sich damit fundamental von jener unabhängiger FotojournalistInnen, die für Medien oder Nachrichtenagenturen tätig sind.

Politische Kommunikation ist jene Kommunikation, die von politischen AkteurInnen ausgeübt wird, an sie gerichtet ist, oder sich auf ihre Aktivitäten bezieht (vgl. Schulz 20II, I6). Visualität zählt zu ihren zentralen Bestandteilen (vgl. Schill 20I2). Visuelle politische Kommunikation ist für die Selbstdarstellung politischer AkteurInnen ebenso relevant wie für die mediale Vermittlung von Politik: „Die Grundlage für die politikwissenschaftliche Forschungsrelevanz im Bereich der visuellen Kommunikation besteht im going public der politischen Akteure, also in der politischen Kommunikation und Informationsverarbeitung in der Medienarena" (Ballensiefen 2009, 38). Dieses going public ist heute in hohem Maße professionalisiert und folgt den Regeln politischen Marketings und politischer PR.

Wenn Politik sich beispielsweise im Rahmen so genannter Photo Opportunities FotojournalistInnen zu sehen gibt, bleibt in der Regel nichts dem Zufall überlassen: „Das beginnt bei der Auswahl des Ortes und des Hintergrundes inklusive Schönwetter- und Schlechtwettervariante, geht über Akkreditierung der Fotojournalisten, die Platzierung der Fotografierten und der Fotografierenden und reicht bis zur Beleuchtung" (Grittmann 2009, 33). Da die Logik visueller Kommunikation auf Assoziationen basiert (Müller 2003, 22), sind Settingwahl und Rollengestaltung für PolitikerInnen entscheidend, um durch visuell verkörperte Eigenschaften politische Images zu beeinflussen (vgl. Graber 1987). „Visual images play a central role in constructing political images (...) they serve as arguments, have an agenda setting function, dramatize policy, aid in emotional appeals, build the candidate's image, create identification, connect to societal symbols, transport the audience, and add ambiguity" (Schill 2012, II8ff.). Dabei geht es vor allem darum, etwas sichtbar zu machen, was zunächst nicht sichtbar ist oder sich sprachlich nur schwer vermitteln ließe (z. B. Führungsstärke).

In besonders intensivem Maß findet eine Strategieverlagerung der visuellen Kommunikation politischer AkteurInnen in Wahlkampfzeiten statt und wird im Rahmen des Forschungsfeldes der Visuellen Wahlkampfkommunikation untersucht (vgl. etwa Müller 1997, Knieper/Müller 2004, Liebhart/Bernhardt 2017). Die strategische Bildverwendung bzw. das Management strategischer Sichtbarkeit beschränkt sich freilich nicht auf Wahlkämpfe. Auch im politischen Alltag nutzen PolitikerInnen Bildmaterial zur Verbreitung ihrer Botschaften: „In politics, managing the public image and reputation of a party and its leader is often intertwined with selling policy, and it has an important endgame given that electors' evaluation of leaders is a key variable in vote choice" (Marland 2012, 216).

Der russische Präsident Wladimir Putin inszeniert sich beispielsweise als sportlich vielseitiger Politiker beim Judo, Schwimmen, Eishockey, Tauchen, Autorennfahren oder Reiten. Die visuellen Anleihen im Feld des Sports dienen dabei als symbolische Bildressource, etwa um Stärke, Disziplin oder Durchhaltevermögen zu signalisieren. Auch die russische Landschaft, die Putin häufig als Kulisse für seine sportlichen Aktivitäten dient, kann als symbolische Botschaft gelesen werden: es handelt sich dabei um eine bestimmte Landschaft (die sibirische Tundra und Taiga), die in der russischen Öffentlichkeit auch historisch mit nationaler Identität verbunden wird. Die dabei entstandenen (offiziellen) Bilder werden in politischen Konflikten auch in den internationalen Medien aktualisiert, etwa wenn Körper und Habitus des Politikers symbolisch als „starker Mann“ gedeutet werden und damit Putins politisches Handeln interpretiert wird (vgl. Foxall 20I3).

In digitalen Öffentlichkeiten ändern sich die Rahmenbedingungen für ein Management strategischer Sichtbarkeit durch politische AkteurInnen (vgl. Bernhardt 20I7). Durch den Bedeutungsgewinn Sozialer Netzwerke für die politische Kommunikation wird Politik nicht mehr nur in der klassischen Medienarena sichtbar, sondern verlagert sich auch ins Internet, wo politische AkteurInnen die Kanäle klassischer Medien umgehen und eigene Geschichten im Sinne eines Storytelling formen können: „Social media enables a type of long-form storytelling with few limitations on content controlled by the candidate" (Page/Duffy 2016, 3). Vor allem SpitzenpolitikerInnen lassen sich von persönlichen FotografInnen begleiten, die sowohl Amtsgeschäfte als auch semi-private Momente festhalten und den Blick auf die politische Persönlichkeit visuell beeinflussen. Die dabei entstandenen Bilder werden als sogenannte "Handout Photos" an Medien weitergegeben oder direkt 
über die offiziellen Social Media Kanäle von PolitikerInnen verbreitet.

Die deutsche Kommunikationswissenschaftlerin Elke Grittmann hat herausgearbeitet, dass sowohl die mediale Selbstdarstellung von Politik als auch ihre mediale Darstellung einem Auswahlprogramm unterliegen, bei dem sich wiederkehrende Bildtypen auf Basis konventionalisierter Bedeutungen erkennen lassen (vgl. Grittmann 2007). Diese Bildtypen können sich auf AkteurInnen, Ereignisse oder Phasen des politischen Prozesses (z.B. Wahlen, Interessenartikulation, etc.) beziehen (Grittmann 2007, 393). Für die Visuelle Politik ergeben sich dadurch Fragen nach Bildstrategien und Darstellungsmerkmalen politischer AkteurInnen, die von den strukturellen Bedingungen des jeweiligen kommunikativen Kontexts beeinflusst werden. Diese Analyse kann sich sowohl auf die Ebene der KommunikatorInnen beziehen und strategisches Bildhandeln von PolitikerInnen untersuchen (vgl. etwa Bernhardt 2017, Bernhardt/Liebhart 2017, Liebhart 2013), als auch die mediale Ebene der Politikvermittlung und ihre spezifischen Regeln und Konventionen in den Blick nehmen (vgl. Mortensen 2015).

Kommen wir noch einmal auf das Foto im State Dining Room zurück: die Autorin Megan Garber beschreibt den Empfang in einem Artikel für das Magazin The Atlantic als „Trumpian strain of spectacle, its images meant to hijack attention and go viral" (Garber 2019). Der Grund für die Aufmerksamkeitslenkung liegt einerseits im Bild selbst und seiner herausragenden kompositorischen Qualität, und andererseits in seiner sprachlichen Rahmung im Zuge der Pressekonferenz. Eine politischikonographische Analyse (vgl. Müller 2OII; Müller/Geise 2015, I83-196) wendet sich zunächst dem Bild selbst zu. Bei der Politischen Ikonografie handelt es sich um einen Forschungsansatz, „der die Ergründung von Bildbedeutungen zum Ziel hat" (Müller 2OII, 29). Im Anschluss an den Kunsthistoriker Erwin Panofsky (1892-I968) baut der Ansatz auf einem Dreischritt unterschiedlicher methodischer Niveaus auf: vorikonografische Beschreibung, ikonografische Analyse und ikonologische Interpretation. Beschreibung und Analyse eines Bildes sind Voraussetzung für seine synthetische Interpretation (vgl. Müller 20II, 29). „Während das Ziel einer ikonografischen Analyse in der systematischen Erfassung, Kategorisierung und Typisierung bestimmter Bildmotive bestehen kann, zielt die ikonologische Interpretation auf die Erfassung der Bedeutungsdimension der untersuchten Bildmotive und der für sie relevanten Produktionsund Rezeptionskontexte" (ebd., 30).

Trump wird auf dem Foto zentriert dargestellt. Er ist formell gekleidet: schwarzer Mantel, schwarze Anzughose, weißes Hemd und rote Krawatte. Trump lächelt. Seine angewinkelten Arme sind geöffnet, sodass seine Handflächen mit leicht gespreizten Fingern zu sehen sind. Er steht im State Dining Room des Weißen Hauses direkt unter einem Portrait von Abraham Lincoln. Die Vorhänge des Raumes sind geschlossen und dieser ist künstlich beleuchtet. Im Hintergrund sind zwei mit bodenlangen Tischtüchern gedeckte Stehtische zu sehen. Im Vordergrund des Fotos befindet sich eine Tafel, auf der goldene Kandelaber und silberne Servierplatten zu sehen sind, auf denen sich in Karton und Plastik verpacktes Fast Food stapelt.

Die Funktion des Raumes (der State Dining Room des Weißen Hauses wird u. a. für Staatsbanketts genutzt) und seine Dekoration lassen darauf schließen, dass es sich um einen Empfang handeln muss. Paradox erscheinen in diesem Zusammenhang nicht nur das verpackte und gestapelte Fast Food (das noch dazu Spitzensportlern serviert wird), sondern auch Trumps Geste, die für die Rolle eines Gastgebers als ungewöhnlich beschrieben werden kann. Durch seine Positionierung im Raum wird Trump mit dem Lincoln-Portrait in Bezug gesetzt. Das Ölgemälde stammt von George P. A. Healy aus dem Jahr 1869. Lincoln ist auf dem Gemälde in der Pose eines Denkers portraitiert, während Trump auf dem Foto als ein "Macher" erscheint. Diese Deutung wird durch die Einbeziehung seiner Wortmeldungen im Zuge der Pressekonferenz und der Photo Opportunity als relevante Kontextinformation verstärkt. Trump betont, dass er für das Fast Food von McDonald's, Wendy's, Burger King und Domino's selbst bezahlt habe. Mit dem Verweis auf "great American food" bedient er nicht nur eine patriotische Erzählung, die an seinen Wahlkampfslogan Make America Great Again anknüpft, sondern legt die Deutung nahe, dass er den Empfang trotz des Shutdowns organisieren konnte und das Government vielleicht doch nicht ganz so wichtig ist, wie immer wieder behauptet wird: I alone can fix it (Garber 2019). Für die politische Bühne sind solche „Strategien der Theatralisierung und Selbstdarstellung wie geschaffen", wie Christian Schicha $(2009,56)$ - unter Verweis auf die schauspielerischen Qualitäten des ehemaligen US-Präsidenten Ronald Reagan - bemerkt. Letzterem wird das Zitat zugeschrieben "A substantial part of the political thing is acting and role playing and I know how to do that" (ebd.). Murray Edelman (I990, zuerst I964 und I97I) hat bereits in den I970er Jahren diese Form der Darstellungspolitik und des dramaturgischen Darstellungshandelns beschrieben und sie von der Herstellungspolitik unterschieden.

Seine Vorliebe für Fast Food betonte Trump bereits während des Wahlkampfes 2016 und inszenierte sich wiederholt beim Genuss von Burgern, Pommes und Hühnerschenkel. In ihrer Studie zu US-Präsidentschaftswahlkämpfen zwischen 1828 und 1996 hat sich die Kommunikations- und Politikwissenschaftlerin Marion G. Müller eingehend mit politischen Bildstrategien beschäftigt (vgl. Müller 1997). Müller beschreibt die Inszenierung von Vorlieben für populäre Speisen oder 
Freizeitbeschäftigungen in der Politik als Elemente einer Common-Man-Strategie, also einer strategischen Selbstpräsentation als Mann bzw. Frau von nebenan: „In der amerikanischen Demokratie muß ein Held immer zugleich auch ein Mann einfacher Herkunft sein und diese nötigenfalls konstruieren, um als Präsidentschaftskandidat wählbar zu werden" (Müller 1997, I87, vgl. auch Seeßlen 20I7). Elementen dieser Inszenierung folgt auch Donald Trump (siehe Abb. 2 und 3): Seht her, ich bin zwar mit meinem Privatjet unterwegs, aber ich habe die gleichen Vorlieben wie ihr!

Abbildung 2: Donald J. Trump an Bord seines Flugzeugs während des Präsidentschaftswahlkampfes am 25.05.2016.
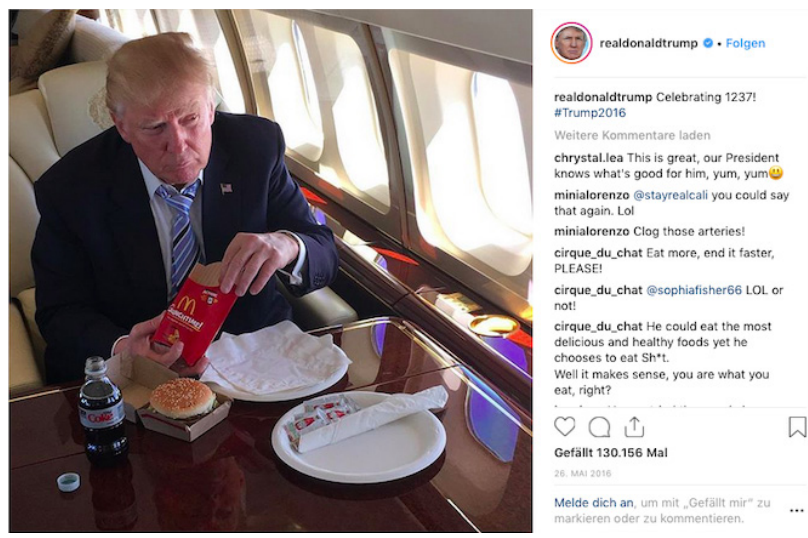

Screenshot eines Instagram-Eintrags von Donald J. Trump, veröffentlicht am 26.05.2016 (https://www.instagram.com/p/BF4raEHmhag/?hl=de)

Der Fast Food Empfang erfüllt für Trump aber noch eine weitere Funktion, die sich auf die Politik seines Amtsvorgängers Barack Obama bzw. der ehemaligen First Lady bezieht. Michelle Obama gründete mit Let's Move! eine Initiative, die sich dem Kampf gegen Übergewicht bei Kindern und dem Einsatz für gesunde Ernährung verschrieben hat. Die ehemalige First Lady nutzte den State Dining Room wiederholt für Auftritte im Zusammenhang mit Let's Move!, unter anderem im Rahmen einer Pressekonferenz mit den Sesame StreetFiguren Elmo und Rosita am 20. Oktober 2013 (Cohen 2013) oder im Rahmen eines Kochevents mit gesundem Gemüse aus dem White House Kitchen Garden am I2. Juni 2014 (Cohen 20I4). In diesem Zusammenhang kann Trumps Fast Food Empfang auch als Versuch einer Überschreibung der Agenda seines Vorgängers bzw. der ehemaligen First Lady interpretiert werden, wie die Bildwissenschaftlerin Cara Finnegan im Rahmen einer Bildbesprechung argumentiert (Chatting the Pictures 2019).
Abbildung 3: Donald J. Trump an Bord seines Flugzeugs während des Präsidentschaftswahlkampfes am 02.08.2016.

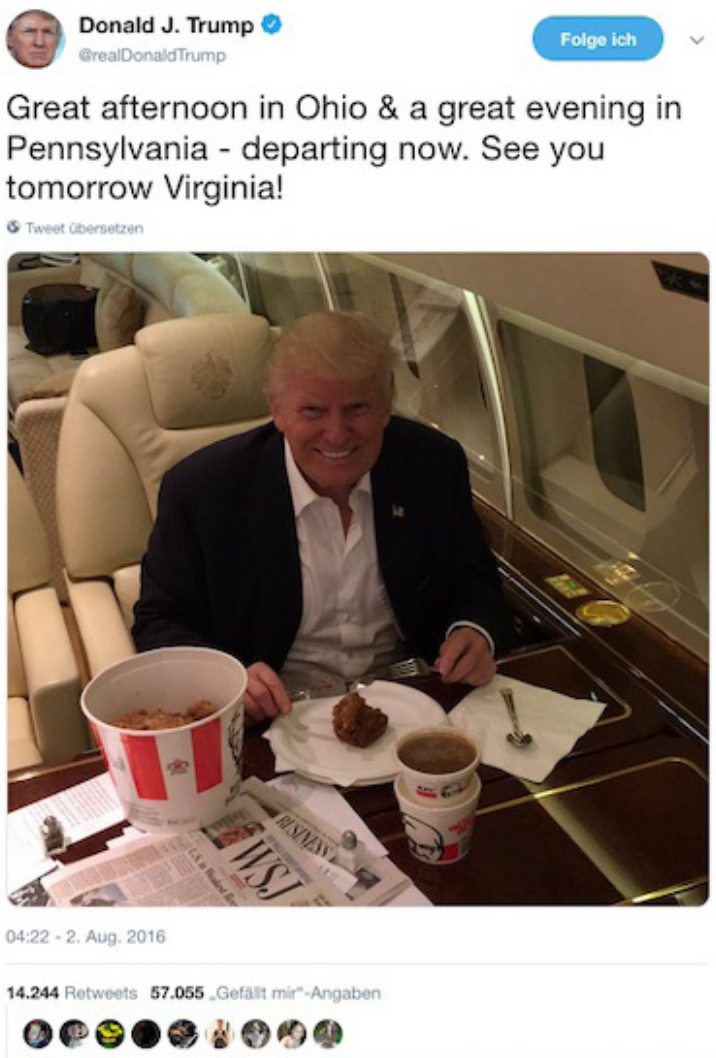

○ 10 Tad. T2 14 Tsd. $\bigcirc 57$ Tsd. $\boxminus$

Screenshot eines Tweets von Donald J. Trump, veröffentlicht am 02.08.2016 (https://twitter.com/realdonaldtrump/status/760299757206208512?lang=de)

\section{Mediale Rezeption und Bildaneignung in Sozialen Netzwerken}

Ein weiterer Aspekt, der das Bildmaterial aus dem State Dining Room als Gegenstand Visueller Politik interessant macht, ist seine breite mediale Rezeption (v.a. von Fotos der Photo Opportunity), seine virale Verbreitung in Sozialen Netzwerken (v.a. des Fotos von Shealah Craighead) und sein Status als politisches Meme (v.a. Bearbeitungen des Fotos von Shealah Craighead). Am Tag nach dem Empfang wurde über das Ereignis in zahlreichen nationalen und internationalen Medien berichtet, was nicht an seinem Nachrichtenwert, sondern vielmehr am dabei entstandenen Bildmaterial liegt. CNN Politics etwa beschrieb das Foto als „Donald Trump's epic fast food picture" (Cillizza 2019), die Neue Zürcher Zeitung (Krapf 2019), die Süddeutsche Zeitung (Klasen 2019), The Guardian (Parkinson 2019) oder The Washington Post (Gebelhoff 2019) berichteten über das Ereignis und ver- 
suchten es mitunter auch einzuordnen: „The reception, no doubt, was an attempt to make the president more relatable, but if anything, his cornucopia of greasy indulgence should serve as a symbol of his presidency" (Gebelhoff 2019).

Auch in Sozialen Netzwerken, wo sich vor allem das offizielle White House Foto von Shealah Craighead viral verbreitete, gab es zahlreiche Reaktionen, die sich das Bild in unterschiedlicher Weise angeeignet haben. Der Medienwissenschaftler Andreas Hepp versteht unter dem Begriff der Aneignung von Bildern einen „sowohl aktiven als auch kulturell umfassenden kontextualisierten Prozess des ,Sich-zu-Eigen-Machens" von Medieninhalten (Hepp 2005, 67). Im Aneignungsprozess spielen zahlreiche Faktoren eine Rolle wie beispielsweise der persönliche Erfahrungshintergrund aber auch Präferenzen und das Wissen von RezipientInnen. Von der Bildaneignung sind Bildhandlungen zu unterscheiden, die sich als „aktive, intendierte Praktiken im Umgang, der Handhabung und Kommunikation mit und durch Bilder" (Müller/Geise 2015, 82) beschreiben lassen. Wenn ein Nutzer oder eine Nutzerin eines Sozialen Netzwerks das Bild bearbeitet, um damit ein Argument zu machen und es an weitere NutzerInnen zu verbreiten, dann ist dies eine Bildhandlung. Diese Bildhandlungen können wiederum Positionen in besonders pointierter Form auf den Punkt bringen und sich zu einem Meme entwickeln. Im Falle des Trump-Fotos ist genau das passiert. Die Plattform Know Your Meme listet es aufgrund seiner Verbreitung bereits als Donald Trump's ,Hamberder Dinner' (Know Your Meme 2019). ${ }^{4}$ Trumps Selbstinszenierung, die mit Konventionen präsidentieller Rollendarstellung bricht, wird somit zu einem Bild, das die projektiven Erwartungen unterschiedlicher Gruppen bedient.

Exemplarisch sei hier auf eine von zahlreichen Reaktionen auf der Mikrobloggingplattform Twitter verwiesen, bei der das Foto aus dem State Dining Room in ironisch-kommentierender Weise bearbeitet wurde (Abb. 4). Im Zuge der Bildbearbeitung wurde der Kopf des US-Präsidenten durch jenen des Clowns Ronald McDonald ersetzt, der das offizielle Maskottchen des Fast-Food-Unternehmens McDonald's darstellt. Der Bildkommentar legt unterschiedliche Interpretationen nahe: zum einen verweist er auf die ungewöhnliche Speisenauswahl, die unter anderem beim genannten Fast-Food-Unternehmen bestellt wurde. Zum anderen thematisiert er die Amtsfähigkeit des US-Präsidenten, der in zahlreichen Kommentaren und Karikaturen als clowneske oder infantile Figur gezeichnet wird.

In ihrem Beitrag für The Atlantic beschreibt Megan Garber das Foto aus dem State Dining Room als typisches Beispiel für virale Ikonografie im digitalen Zeit-

4 Die Bezeichnung Hamberder leitet sich aus einem Schreibfehler Trumps in einem Tweet ab.
Abbildung 4: Reaktion eines Twitter-Users auf das Foto aus dem State Dining Room
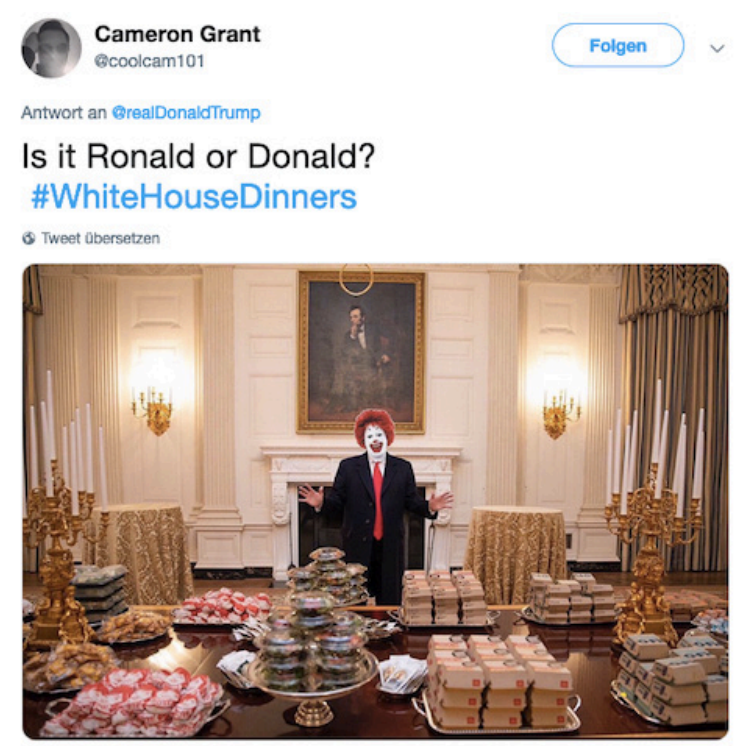

13:17 - 15. Jan. 2019

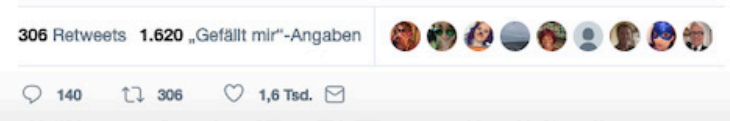

Screenshot eines Tweets des Users Cameron Grant, veröffentlicht am 15.01.2019 (https://twitter.com/realdonaldtrump/status/760299757206208512?lang=de)

alter. Die Transformation des Fotos in ein Meme durch seine massenhafte Bearbeitung und Weiterverbreitung verdeutlicht die dem Meme inhärente Lust am Storytelling, also am Geschichtenerzählen über und durch Bilder (Garber 2019). Sowohl Trump-BefürworterInnen als auch Trump-GegnerInnen sehen sich durch das Foto in ihren Annahmen über den Präsidenten und seine Amtsführung bestätigt. Trump sei sich dieser Wirksamkeit durchaus bewusst und bediene "memes as tools of argument" (ebd.).

\section{Zusammenfassung und Ausblick: Visuelle Kompetenz für die Politikwissenschaft}

Dieser Beitrag hat Visualität als eine zentrale Komponente politischen Handelns und politischer Kommunikation benannt und für eine intensivere Beschäftigung mit visuellen Phänomenen im Rahmen politikwissenschaftlicher Forschungsdesigns plädiert. ${ }^{5}$ Um den thematischen Zugang zu erleichtern und eine fachinterne

5 Ausführlicher und mit einer Reihe weiterer Beispiele dazu auch das Sonderheft der Österreichischen Zeitschrift für Soziologie (2/2016) zum Thema „Bildhandeln und Visuelle Politik”, das von Roswitha Breckner und Andreas Pribersky herausgegeben wurde (vgl. Breckner/ Pribersky 2016). 
Diskussion anzuregen, wurden Prämissen des interdisziplinär ausgerichteten Forschungsbereichs Visuelle Politik vorgestellt. Die Diskussion unterschiedlicher Fragestellungen und Analyseperspektiven erfolgte auf Basis eines aktuellen Fotos von Donald J. Trump, das dem Produktionskontext strategischer politischer Kommunikation entstammt und eine breite Rezeption in klassischen Medien und Sozialen Netzwerken erfahren hat. Entlang des Fotos wurde gezeigt, wie die Politikwissenschaft durch die Entwicklung visueller Kompetenz (vgl. Müller 2008; Müller/Geise 2015, I77ff.) profitieren kann. Eine visuell orientierte Politikwissenschaft zeichnet sich durch eine prinzipielle Offenheit gegenüber Forschungsfeldern aus, in denen eine Beschäftigung mit dem Medium Bild methodisch und theoretisch vorangetrieben wird. Diese Offenheit erschließt nicht bloß kulturwissenschaftliche Ansätze und Methoden zur Analyse sozialer Phänomene: Sie eröffnet damit auch eine Verbindung zur aktuellen Diskussion von Visualität als einem zentralen anthropologischen Merkmal, die eine Verbindung zu naturwissenschaftlichen Untersuchungen der Rolle visueller Wahrnehmung für Gedächtnis und Bewußtsein herstellt (vgl. Forster 2018, I39ff und Kandel 2006). Grundlage dafür ist eine Anerkennung der spezifischen assoziativen Logik von Bildern, die sich von der argumentativen Logik in Textkommunikation wesentlich unterscheidet (Müller/Geise 2015, 37). Bilder beschreiben nicht, sie "veranschaulichen” einen Sachverhalt oder ein Phänomen. Bilder sind zwar selten völlig „sprachfrei” (wir verständigen uns sprechend über sie), jedoch kann Sprache nicht alle Aspekte der Wahrnehmung erfassen (vgl. Grittmann 2007). ${ }^{6}$ Dabei behält eine visuell orientierte Politikwissenschaft im Blick, dass Visuelle Kommunikation kaum je isoliert auftritt, sondern zumeist in Verbindung mit anderen kommunikativen Modi zu untersuchen ist. Nicht zuletzt setzt sich eine visuell orientierte Politikwissenschaft mit den je spezifischen Entstehungs-, Verbreitungs-, Aneignungsund Rezeptionsbedingungen politischer Bilder auseinander.

Marion G. Müllers Überlegungen zu visueller Kompetenz (vgl. z. B. Müller 2008; Müller/Geise 2015) im Sinne einer grundlegenden bildspezifischen Kompetenz sind in diesem Zusammenhang auch für die politikwissenschaftliche Forschung gewinnbringend: Das Konzept „Visuelle Kompetenz” bezeichnet ein „idealtypisches Denkmodell zur Strukturierung komplexer Fragestellungen der Visuellen Kommunikationsforschung" (Müller/Geise 2015, I82) und dient sowohl der theoretischen

6 Als ein Beispiel kann hier die Analyse visueller Metaphern bzw. visueller Repräsentationen metaphorischer Konzepte angeführt werden. Zwar sind die Grenzen zwischen sprachlichen und visuellen Metaphern fließend, da beide letztlich auf mentale Bilder rekurrieren. Visuelle Metaphern als bildlicher Ausdruck einer Bedeutungsübertragung erfordern aber bildspezifische Analysemethoden, die Motive und deren Bedeutungstraditionen in den Blick nehmen. als auch der empirischen Forschung (vgl. ebd.). Der Begriff der Kompetenz ist als „übergeordnete Kategorie” (Müller/Geise 2015, 178) zu verstehen, die die Herstellung (Produktion), die Wahrnehmung (Perzeption) und die Bedeutungszuweisung (Interpretation) von Bildern sowie die kognitiv-emotionale Verarbeitung bildlicher Eindrücke (Rezeption) umfasst. Visueller Kompetenzforschung geht es nicht primär darum, einen Forschungsbereich um eine visuelle Dimension zu erweitern (ebd.), sondern vielmehr um eine grundsätzliche transdisziplinäre Orientierung, die mit einer "andere[n] Analyse- und Interpretationskompetenz als dies für Textkommunikation der Fall ist" (Müller/Geise 2015, I77) verbunden ist.

Die Analyse politischer Bilder erfordert deshalb auch den Einsatz geeigneter methodischer Instrumentarien. Methodische Zugänge, die für die Analyse von Texten entwickelt wurden (wie etwa quantitative oder qualitative inhaltsanalytische Verfahren, aber auch Ansätze aus dem vielfältigen Spektrum diskursanalytischer $\mathrm{Zu}$ gänge), reichen letztlich nicht aus, um politische Bilderwelten zu analysieren. Eine bildkompetente Politikwissenschaft, die Fotos nicht „als ,Abbild' einer wie immer gearteten ,Realität" (Matjan 2002, I88) betrachtet, sondern sich mit der „Untersuchung des Spannungsfeldes zwischen dem Bildinhalt, seiner symbolischen Bedeutung und seinem Erscheinungs- und Wahrnehmungskontext" (ebd.) befasst, könnte sich mit der politischen Funktion vor allem (aber nicht ausschließlich) fotografischer Bilder aus unterschiedlichen Perspektiven beschäftigen:

I. Analysen der je spezifischen Produktionsbedingungen politischer Bilder und Diskussion der damit verbundenen Implikationen (z. B. strategischer Produktionskontext versus journalistischer Produktionskontext) mit der Methode der Visuellen Kontextanalyse (vgl. Müller/Geise 20I5). Die Aufnahmen aus dem State Dining Room machen deutlich, dass persönliche FotografInnen und JournalistInnen bisweilen unterschiedliche Perspektiven auf ein Ereignis nehmen und dass sich diese Perspektiven auf Wirkungspotenziale des Bildmaterials auswirken können.

2. Analysen sich wiederholender Bildmotive und Darstellungskonventionen sowie Rekonstruktion von Bedeutungstraditionen, z. B. mit der Methode der Quantitativen Bildtypenanalyse (vgl. etwa Grittmann/ Ammann 20II) oder der Politischen Ikonografie (vgl. Müller 20II, Müller/Geise 20I5). Das Foto aus dem State Dining Room erscheint in dieser Perspektive als gezielter Bruch mit den motivischen Konventionen eines politischen Empfangs, der dazu dient, Aufmerksamkeit zu bündeln und über die Logik der Darstellung Botschaften über den US-Präsidenten und sein Amtsverständnis zu vermitteln. 
3. Analysen unterschiedlicher medialer Kontextualisierungen politischer Bilder und Rekonstruktion unterschiedlicher Interpretationen (z. B. mit der Methode der Visuellen Kontextanalyse (vgl. Müller/Geise 2015) oder der Visuellen Frame-Analyse (vgl. etwa Geise/Lobinger 20I3). Das Bildmaterial aus dem State Dining Room wird in dieser Perspektive in seinem medialen Verwendungszusammenhang thematisiert, der mit unterschiedlichen Bedeutungszuweisungen und -mustern verbunden sein kann.

4. Analysen der Aneignungsformen politischer Bilder in Sozialen Netzwerken und Rekonstruktion unterschiedlicher Deutungszuschreibungen durch NutzerInnen, etwa mit der Methode der Visuellen Frame-Analyse (vgl. Geise/Lobinger 20I3). Hier richtet sich der Blick auf die soziale Gebrauchsweise des Fotos durch Publikumsgruppen, die es in ihre Kommunikationsakte einbetten (ggf. in bearbeiteter, kommentierender Form).

Diese Ansätze ermöglichen einen multiperspektivischen Blick auf politisches Bildmaterial und seine Funktionen im Rahmen strategischer politischer Kommunikation, politischer Auseinandersetzungen sowie im Rahmen öffentlicher und medialer Debatten. Das Trumpsche Spektakel, wie es im vorliegenden Beitrag diskutiert wurde, wird dadurch als strategischer Bildeinsatz in einem hybriden Mediensystem (vgl. Chadwick 20I3) analysierbar, der die Logiken medialer Aufmerksamkeit für sich zu nutzen weiß, um politische Kommunikations- und Identifikationsangebote nicht nur sprachlich, sondern auch visuell zu verankern.

\section{Literatur}

Ballensiefen, Moritz (2009), Bilder machen Sieger - Sieger machen Bilder: Die Funktion von Pressefotos im Bundestagswahlkampf 2005, Wiesbaden: VS Verlag.

Bernhardt, Petra (20I7), Image-Making - Image Management: White House Photos and the Political Iconography of the Obama Presidency, in: The Poster, Vol. 4(I-2), I45-I72.

Bernhardt, Petra/Karin Liebhart (20I7), Politik auf Instagram: Bildstrategien von Norbert Hofer und Alexander Van der Bellen im Bundespräsidentschaftswahlkampf 2016, in: SWS Rundschau, Vol. 57(2), I46-167.

Beyme, Klaus von (1987), Der Wiederaufbau: Architektur und Städtebaupolitik in beiden deutschen Staaten, München: Piper.

Beyme, Klaus von (2005), Das Zeitalter der Avantgarden: Kunst und Gesellschaft 1905-1955, München: C.H. Beck

Bleiker, Roland (20I8) (Hg.), Visual Global Politics, London/New York: Routledge/Taylor \& Francis Group.
Boehm, Gottfried (I994), Was ist ein Bild? München: Fink.

Boehm, Gottfried (2007), Wie Bilder Sinn erzeugen: Die Macht des Zeigens, Berlin: Berlin University Press.

Breckner, Roswitha/Andreas Pribersky (Hg.) (20I6), Bildhandeln und Visuelle Politik, Österreichische Zeitschrift für Soziologie, 2/2016.

Burke, Peter (1992), The Fabrication of Louis XIV, New Haven: Yale University Press.

Chadwick, Andrew (2013), The hybrid media system: Politics and power, Oxford: Oxford University Press.

Chatting the Pictures (2019), Race And Trump's Fast Food Feast at the White House, Internet: https://www.readingthepictures.org/2019/or/race-trump-fast-foodfeast/ (Zugriff: 20.02.2019).

Cillizza, Chris (2019), Donald Trump's epic fast food picture is perfectly Trumpian, Internet: https://edition.cnn.com/2019/or/I5/politics/donald-trumpfast-food-clemson-tigers/index.html (Zugriff: 20.02.2019).

Cohen, Elyse (20I3), Elmo and Rosita Join First Lady Michelle Obama to Promote Fresh Food Choices for Kids, Internet: https:/obamawhitehouse.archives. gov/blog/2OI3/IO/3I/elmo-and-rosita-join-firstlady-michelle-obama-promote-fresh-food-choiceskids (Zugriff: 20.02.2019).

Cohen, Elyse (2014), From White House Kitchen Garden to State Dining Room: The 2014 Garden Harvest, Internet: https:/letsmove.obamawhitehouse.archives. gov/blog/2OI4/06/I3/white-house-kitchen-gardenstate-dining-room-2oI4-garden-harvest (Zugriff: 20.02.2019).

Dörner, Andreas (200I), Politainment: Politik in der medialen Erlebnisgesellschaft, Frankfurt am Main: Suhrkamp.

Drechsel, Benjamin (2007), Was ist ein politisches Bild? Einige Überlegungen zur Entwicklung der Politikwissenschaft als Bildwissenschaft, in: Mitterbauer, Helga/Ulrich Tragatschnig (Hg.): Kulturwissenschaftliches Jahrbuch Moderne (Themenschwerpunkt: Iconic Turn), Innsbruck: Studienverlag, IO6-I2O.

Drechsel, Benjamin (2009), Trügerischer Augenschein? Hinweise zur Verflechtung von politischer Kultur und visueller Politik, in: Salzborn, Samuel (Hg.) (2009), Politische Kultur. Forschungsstand und Forschungsperspektiven, Frankfurt am Main: Peter Lang, I47-I74.

Edelman, Murray (1990), Politik als Ritual: Die symbolische Funktion staatlicher Institutionen und politischen Handelns (Originalausgabe gesondert 1964 und 197I), Frankfurt am Main/New York: Campus.

Forster, Kurt W. (2018), Aby Warburgs Kulturwissenschaft: Ein Blick in die Abgründe der Bilder, Berlin: Matthes \& Seitz. 
Foxall, Andrew (2013), Photographing Vladimir Putin: Masculinity, Nationalism and Visuality in Russian Political Culture, in: Geopolitics, Vol. I8(I), I32-56.

Garber, Megan (2019), The President's McFeast, Internet: https://www.theatlantic.com/entertainment/ archive/2019/oI/the-political-logic-of-trumpsfast-supper/58050I/?utm_medium=social\&utm content=edit-promo\&utm_campaign $=$ the atlantic\&utm_term=20I9-OI-I5T2 I \% 3 A 34 \%3A34\&utm_source=twitter (Zugriff: I6.0I.2019).

Gebelhoff, Robert (2019), Donald Trump's fast-food presidency, Internet: https://www.washingtonpost. com/opinions/2019/or/15/donald-trumps-fast-foodpresidency/?utm_term=.d6ec68a61664 (Zugriff: 20.02.2019).

Geise, Stephanie/Katharina Lobinger (Hg.) (2013), Visual Framing: Perspektiven und Herausforderungen der Visuellen Kommunikationsforschung, Köln: Herbert von Halem Verlag.

Graber, Doris A. (1987), Kind words and harsh pictures: How television presents the candidates, in: Schlozman, Kay Lehmann (Hg.), Elections in America, Winchester, MA: Allen \& Unwin, II5-I4I.

Grittmann, Elke (2007), Das politische Bild: Fotojournalismus und Pressefotografie in Theorie und Empirie, Köln: Herbert von Halem Verlag.

Grittmann, Elke (2009), Das Bild von Politik: Vom Verschwinden des entscheidenden Moments, in: Aus Politik und Zeitgeschichte, Vol. 31, 33-38.

Grittmann, Elke/Ilona Ammann (2OII), Quantitative Bildtypenanalyse, in: Petersen, Thomas/Clemens Schwender (Hg.), Die Entschlüsselung der Bilder: Methoden zur Erforschung visueller Kommunikation, Köln: Herbert von Halem Verlag, I63-I78.

Hepp, Andreas (2005), Medienkultur, in: Hepp, Andreas/ Friedrich Krotz/Carsten Winter (Hg.), Globalisierung der Medienkommunikation: Eine Einführung, Wiesbaden: VS Verlag, I37-I62.

Hofmann, Wilhelm (20II) (Hg.), Stadt als Erfahrungsraum der Politik: Beiträge zur kulturellen Konstruktion urbaner Politik, Berlin: Lit.

Jarren, Otfried/Patrick Donges (2OII), Politische Kommunikation in der Mediengesellschaft: eine Einführung, Wiesbaden: VS Verlag für Sozialwissenschaften.

Kandel, Eric R. (2006), In Search of Memory: The Emergence of a New Science of Mind, New York: Norton.

Klasen, Oliver (2019), Royal mit Käse, Internet: https://www.sueddeutsche.de/panorama/trump-fastfood-burger-weisses-haus-I.428827I

(Zugriff: 20.02.2019).

Knieper, Thomas/Marion G. Müller (Hg.) (2004), Visuelle Wahlkampfkommunikation, Köln: Herbert von Halem Verlag.

Know Your Meme (2019), Donald Trump's „Hamberder” Dinner, Internet: https://knowyourmeme.com/me- mes/events/donald-trumps-hamberder-dinner ( $\mathrm{Zu}-$ griff: 20.2.2019).

Krapf, Christof (2019), Donald Trump setzt dem Meister im College-Football Fast Food vor, Internet: https://www.nzz.ch/sport/donald-trump-setztdem-meister-im-college-football-beim-empfangim-weissen-haus-fast-food-vor-ld.I451513 (Zugriff: 20.02.2019).

Liebhart, Karin (2013), Icon of the Orange Revolution, Evita of Kyiv, Ukrainian Barbie doll, Jeanne d'Arc, innocent victim? The multifaceted visual representations of Yulia Tymoshenko, in: Politics in Central Europe, Vol. 9(I), 27-46.

Liebhart, Karin/Petra Bernhardt (2017), Political Storytelling on Instagram: Key Aspects of Alexander Van der Bellen's Successful 2016 Presidential Election Campaign, in: Media and Communication, Vol. 5(4), I525.

Lobinger, Katharina (2015), Visualität, in Hepp, Andreas/ Friedrich Krotz/Swantje Lingenberg/Jeffrey Wimmer (Hg.), Handbuch Cultural Studies und Medienanalyse, Wiesbaden: VS Verlag, 9I-Ioo.

Marland, Alex (2012), Political Photography, Journalism, and Framing in the Digital Age: The Management of Visual Media by the Prime Minister of Canada, in: The International Journal of Press/Politics, Vol. I7(2), 214233.

Matjan, Gregor (2002), Wenn Fotos nicht lügen können, was können sie dann? Zum Einsatz der Fotoanalyse in der Politikwissenschaft, in: Österreichische Zeitschrift für Politikwissenschaft, Vol. 3I(2), I73-190.

Meyer, Thomas (200I), Mediokratie: Die Kolonisierung der Politik durch die Medien, Frankfurt am Main: Suhrkamp.

Meyer, Thomas (2010), Was ist Politik? Wiesbaden: Springer.

Mitchell, William J.T. (1990), Was ist ein Bild? In: Bohn, Volker (Hg.), Bildlichkeit. Internationale Beiträge zur Poetik, Frankfurt am Main: Suhrkamp, 17-68.

Mitchell, William J.T. (1992), The Pictorial Turn, in: Art Forum International, Vol. 30(3), 89-94.

Mortensen, Tara (2015), Visually Assessing the First Lady in a Digital Age: A Study of Michelle Obama as Portrayed by Journalists and the White House, in: Journal of Women, Politics \& Policy, Vol. 36(I), 43-67.

Müller, Marion G. (1997), Politische Bildstrategien im amerikanischen Präsidentschaftswahlkampf, I8281996, Berlin: Akademie Verlag.

Müller, Marion G. (2003), Grundlagen der visuellen Kommunikation: Theorieansätze und Analysemethoden, Konstanz: UTB.

Müller, Marion G. (2008), Visual Competence: A New Paradigm for Studying Visuals in the Social Sciences?, in: Visual Studies, Vol. 23(2), IOI-II2. 
Müller, Marion G. (20II), Ikonografie und Ikonologie, visuelle Kontextanalyse, visuelles Framing, in: Petersen, Thomas/Clemens Schwender (Hg.) (2OII), Die Entschlüsselung der Bilder: Methoden zur Erforschung visueller Kommunikation, Köln: Herbert von Halem Verlag, 28-55.

Müller, Marion G./Stephanie Geise (2015), Grundlagen der Visuellen Kommunikation (2. , überarbeitete Auflage), Konstanz und München: UVK.

Münkler, Herfried/Jens Hacke (Hg.) (2009), Strategien der Visualisierung: Verbildlichung als Mittel politischer Kommunikation, Frankfurt am Main: Campus.

Page, Janis Teruggi/Margaret E. Duffy (2016), What Does Credibility Look like? Tweets and Walls in U.S. Presidential Candidates' Visual Storytelling, in: Journal of Political Marketing, 6. April 2016, I-29, https://doi.org/I o.I080/15377857.2016.1171819.

Parkinson, Hannah Jane (2019), Donald Trump's fast food banquet - feeding the people Whoppers, as ever, Internet: https://www.theguardian.com/commentisfree/20I9/jan/I5/donald-trump-fast-foodwhoppers-president-college-football (Zugriff: 20.02.2019).

Pribersky, Andreas (20II), Die Planstadt als Politische Utopie im 20. Jahrhundert zwischen Kapitalismus und Sozialismus, in: Wilhelm Hofmann (Hg.) (2OII), Stadt als Erfahrungsraum in der Politik, Berlin: LIT, I33-I4I.

Riedl, Andreas/Peter Maurer/Birgit Mühl (20I8), Anpassung an die Medienlogik? Eine Untersuchung politischer Kommunikationsstrategien von AkteurInnen aus Deutschland und Österreich, in: Österreichische Zeitschrift für Politikwissenschaft, Vol. 47(I), I-I6.

Sanders, Sarah (2019), Tweet von @PressSec am I5.OI.20I9, Internet: https://twitter.com/PressSec/ status/I084980942870II840I (Zugriff: I6.oI.20I9).

Seeßlen, Georg (20I7), TRUMP! Populismus als Politik, Berlin: Bertz+Fischer.

Schicha, Christian (2009), Politikvermittlung als legitimes Theater? Die Zuwanderungsdebatte im Bundesrat als Bühnenstück für die Medienöffentlichkeit, in: Herbert Willems (Hg.), Theatralisierung der Gesellschaft (Band 2: Medientheatralität und Medientheatralisierung), Wiesbaden: VS Verlag für Sozialwissenschaften, 53-79.

Schill, Dan (2012), The Visual Image and the Political Image: A Review of Visual Communication Research in the Field of Political Communication, in: The Review of Communication, Vol. I2(2), II8-I42.

Schulz, Winfried (2OII), Politische Kommunikation. Theoretische Ansätze und Ergebnisse empirischer Forschung (3., überarbeitete Auflage), Wiesbaden: VS Verlag.

Sternberger, Dolf (1957), Über den Jugendstil und andere Essays, Hamburg: Claassen.
Sternberger, Dolf (1980), Herrschaft und Vereinbarung, Frankfurt am Main: Suhrkamp.

Strömbäck, Jesper (2008), Four phases of mediatization: An analysis of mediatization of politics, in: The International Journal of Press/Politics, Vol. I3(3), 228-246.

The White House (2019a), Tweet am 15.01.2019, Internet: https://twitter.com/WhiteHouse/status/I08498 829904I738752 (Zugriff: I6.OI.2019).

The White House (2019b), Facebook-Eintrag am I5.0I.20I9, Internet: https://www.facebook.com/WhiteHouse/ videos/Ior66I4055204384/ (Zugriff: I6.oI.20I9).

\section{AutorInnen}

Dr. Petra Bernhardt ist wissenschaftliche Projektmitarbeiterin und Lektorin am Institut für Politikwissenschaft der Universität Wien. Sie beschäftigt sich mit Visueller Politischer Kommunikation, Visuellen Methoden, Fotografie und politischer Bildverwendung in digitalen Öffentlichkeiten. Kontakt: Institut für Politikwissenschaft der Universität Wien, Universitätsstrasse 7/2, IoIo Wien, petra.bernhardt@univie.ac.at.

PD Dr. Karin Liebhart ist Senior Lecturer am Institut für Politikwissenschaft der Universität Wien und Sociology Associate Professor an der Universität Trnava. Ihre Forschungsschwerpunkte sind Visuelle und Diskursive Repräsentationen des Politischen, Visuelle Politische Kommunikation in traditionellen und sozialen Medien, Visuelle Methoden, Rechtspopulismus und Rechtsextremismus. Kontakt: Institut für Politikwissenschaft der Universität Wien, Universitätsstrasse 7/2, IOIO Wien, karin.liebhart@univie.ac.at.

OR Dr. Andreas Pribersky ist wissenschaftlicher Beamter am Institut für Politikwissenschaft der Universität Wien. Forschungsschwerpunkte Politische Ikonografie, Politische Architektur, Ikonoklasmus, Politische Symbole und Rituale, mit einem regionalen Fokus auf Ostmitteleuropa. Kontakt: Institut für Politikwissenschaft der Universität Wien, Universitätsstrasse 7/2, IOIO Wien, andreas.pribersky@univie.ac.at 